Arq. Bras. Med. Vet. Zootec., v.60, n.3, p.536-542, 2008

\title{
Estudo eletrocardiográfico de eqüinos da raça Mangalarga Marchador
}

[Eletrocardiographic study in horses of the Mangalarga Marchador breed]

\author{
M.P. Diniz ${ }^{1}$, R.A.L. $M u z z i^{2}$, L.A.L. $M u z z i^{2}$, G.E.S. Alves $^{3}$ \\ ${ }^{1}$ Aluna de pós-graduação - FMVZ-USP - São Paulo, SP \\ ${ }^{2}$ Departamento de Medicina Veterinária - UFLA - Lavras, MG \\ ${ }^{3}$ Escola de Veterinária - UFMG - Belo Horizonte, MG
}

\begin{abstract}
RESUMO
Avaliou-se o perfil eletrocardiográfico de 60 eqüinos da raça Mangalarga Marchador, clinicamente sadios, com idades variando de um a 28 anos, sendo 39 fêmeas e 21 machos. A freqüência cardíaca tendeu a diminuir com a idade e a amplitude da onda $\mathrm{P}$ aumentou ao longo do crescimento do animal. Os parâmetros de duração de QRS e amplitude de $\mathrm{S}$ foram maiores em machos que em fêmeas.
\end{abstract}

Palavras-chave: eqüino, eletrocardiograma, Mangalarga Marchador

\begin{abstract}
For the electrocardiographic evaluation of Mangalarga Marchador breed horses, 60 electrocardiograms were recorded from clinically healthy equines aging from one to 28-year-old, being 39 females and 21 males. The heart rate tended to decrease with age and the amplitude of $P$ wave increased with the growth of the animals. The duration of the QRS complex and the amplitude of $S$ wave were greater in males than in females.
\end{abstract}

Keywords: horse, electrocardiogram, Mangalarga Marchador breed

\section{INTRODUÇÃO}

O eletrocardiograma (ECG) é um importante método diagnóstico, desde que associado a um exame clínico cardiovascular criterioso, no reconhecimento dos distúrbios de formação e condução do impulso cardíaco, podendo diagnosticar as diversas arritmias, bem como avaliar a evolução de determinadas doenças cardíacas (White e Rhode, 1974). O ECG também tem sido utilizado para predizer performance atlética (Stewart, 1981; Illera e Illera, 1987) e para acompanhar a evolução dos treinamentos físicos e seus excessos (Rose e Davis, 1978). Como foi verificado por Hilwig (1977) e Illera et al. (1987), os parâmetros eletrocardiográficos apresentaram modificações entre as diferentes raças eqüinas, sendo necessário que cada raça tenha seu padrão específico. Ainda, segundo Ayala et al. (1995) e Fernandes et al. (2004), podem existir diferenças entre os parâmetros eletrocardiográficos dos eqüinos ao longo de seu crescimento e em cada raça.

O sistema de derivação bipolar ápice-base é um dos sistemas utilizados em eqüinos que apresentam grande praticidade pelo local de colocação dos eletrodos, além de apresentar traçados regulares com complexos de grande amplitude, que desse modo, facilitam a leitura (Ayala et al., 1994; Patteson, 1996).

Eqüinos saudáveis apresentam incidência de arritmias cardíacas em torno de $25 \%$ a $30 \%$ (Robertson, 1992), sendo várias dessas consideradas fisiológicas. Entretanto, existem arritmias que podem levar à baixa condição

Recebido em 21 de maio de 2007

Aceito em 18 de março de 2008

Endereço para correspondência (corresponding address)

Rua Álvaro Moreira, 355 - 22630-160 - Rio de Janeiro, RJ

E-mail: mpdiniz@usp.br 
física (Mitten, 1996). Algumas podem ser reconhecidas pela auscultação, porém só podem ser realmente confirmadas pelo ECG (Belgrave, 1990).

A raça Mangalarga Marchador é brasileira e tem como principal função a marcha. Os animais apresentam temperamento ativo e dócil, resistência e rusticidade, por isso foram difundidos por todo o País, sendo utilizados em diversas modalidades esportivas.

Considerando que, ao longo do tempo, os eqüinos passaram por constantes adaptações orgânicas e que o funcionamento cardiovascular pode variar segundo as características e as funções impostas às diferentes raças, o presente estudo teve como objetivos descrever o perfil eletrocardiográfico de eqüinos da raça Mangalarga Marchador, comparando-o com os padrões atuais, e estudar a influência do sexo e da idade sobre o eletrocardiograma.

\section{MATERIAL E MÉTODOS}

Foram estudados 60 eqüinos da raça Mangalarga Marchador, 21 machos (35\%) e 39 fêmeas (65\%), com idades entre um e 28 anos. Os animais foram distribuídos em três grupos, de acordo com a idade: grupo 1, formado por animais de até três anos $(\mathrm{n}=17)$; grupo 2, animais com mais de três e até 10 anos $(\mathrm{n}=35)$ e grupo 3 , animais com mais de 10 anos $(n=8)$. O escore corporal foi classificado em uma escala de um a cinco, como descrito por Speirs (1997) e todos os animais tiveram escore corporal três ou quatro.

O critério de exclusão foi a presença de qualquer distúrbio de sanidade, incluindo cardiopatias, verificado por exame clínico geral e específico. $\mathrm{O}$ exame eletrocardiográfico foi realizado sem qualquer tranqüilização, com o animal contido em piso de borracha e em posição ortostática, com os membros paralelos entre si. A distribuição dos eletrodos seguiu o sistema de derivação bipolar ápice-base, descrito por Patteson (1996), posicionando-se o eletrodo amarelo no lado esquerdo acima do ápice cardíaco, logo atrás do olécrano e o eletrodo vermelho no lado direito, cranial à escápula, próximo à veia jugular. Após uso de gel condutor, os eletrodos foram conectados à pele por meio de condutores metálicos tipo jacaré.
Para o exame eletrocardiográfico, utilizou-se um aparelho portátil Funbec Ecafix ECG 40, composto por seis canais, três sensibilidades e velocidade de $25 \mathrm{~mm} / \mathrm{s}$, ligado a um estabilizador de voltagem. O aparelho, regulado para a velocidade de $25 \mathrm{~mm} / \mathrm{s}$ e na sensibilidade de $1 \mathrm{~cm}=1 \mathrm{mV}$, foi registrado na derivação I. Foram coletados, pelo menos, 10 complexos em cada eletrocardiograma.

O estudo dos traçados compreendeu a medida simples da amplitude das ondas em milivolts, a medida da duração das ondas e intervalos em segundos e a análise morfológica das ondas. Determinaram-se, ainda, a freqüência cardíaca, em batimentos por minuto (bpm) e o ritmo cardíaco.

Os resultados foram analisados por meio de cálculos da média e do erro-padrão da média, para as variáveis numéricas de amplitude e duração das ondas, complexos e intervalos e, ainda, freqüência cardíaca. Os cálculos foram feitos por meio do programa estatístico Epi info $^{\mathrm{TM} 1}$. Os resultados foram submetidos à análise de variância $(P \leq 0,05)$ e aos testes $t$ e Tukey para comparação de médias.

\section{RESULTADOS E DISCUSSÃO}

O ritmo mais freqüente encontrado foi o sinusal, com $61,7 \%$ dos casos, seguido de arritmia sinusal, com $28,2 \%$, taquicardia sinusal $(6,7 \%)$, bradicardia sinusal $(1,7 \%)$ e bloqueio sinusal/sinus arrest (1,7\%). Constataram-se, ainda, $1,7 \%$ de bloqueio atrioventricular de segundo grau (BAV $2^{\circ}$ ) e $6,7 \%$ de marcapasso migratório. $\mathrm{O}$ ritmo sinusal, segundo a literatura, é o mais freqüentemente encontrado em eqüinos (Patteson, 1996; Fernandes et al., 2004), porém, a arritmia sinusal, diferente do encontrado por Vincenzi (1995) em eqüinos Mangalarga, esteve mais presente e o marcapasso migratório, menos presente - arritmia sinusal $13,3 \%$ e marcapasso migratório, 20\%. Assim, pode-se considerá-lo como um achado clínico para a amostra estudada, uma vez que os animais não se encontravam em treinamento físico durante $\mathrm{o}$ estudo.

A arritmia sinusal, segundo Patteson (1996), está associada a mudanças no tônus vagal e apresenta

${ }^{1}$ Epi Info Software, versão 3.3.2, 2004 
baixa freqüência em eqüinos em repouso, sendo mais comum aparecer durante a fase de recuperação pós-exercício, particularmente após exercícios leves, pois, nesta situação, quando a freqüência cardíaca diminui, o ritmo volta ao normal. Esses conceitos foram contestados por estudos realizados com a monitoração por holter, nos quais se observam períodos de arritmia sinusal na maioria dos animais (Raekallio, 1992; Scheffer et al., 1995). No presente estudo, observou-se porcentagem elevada de arritmia sinusal em animais com repouso, 28,3\%. A provável explicação para essa arritmia seria a variação no sistema nervoso autônomo, associada à condição física desses animais que não são atletas e não se encontravam em treinamento físico.
$\mathrm{O}$ BAV de $2^{\circ}$ grau, o marcapasso migratório e a bradicardia sinusal foram encontrados em baixa freqüência, sendo um achado clínico. Segundo Mitten (1996), essas são arritmias consideradas fisiológicas para a espécie eqüina, sendo abolidas pelo exercício e/ou estresse. O BAV de $2^{\circ}$ grau foi observado em apenas 1,7\% dos animais, apesar de alguns estudos relatarem que essa é a arritmia mais encontrada em eqüinos, podendo ser observada, durante o repouso, em mais de $30 \%$ dos eqüinos condicionados (Robertson, 1992). A baixa freqüência desta arritmia pode ser explicada pelo fato de os animais estudados não estarem sendo condicionados fisicamente (Fig. 1).

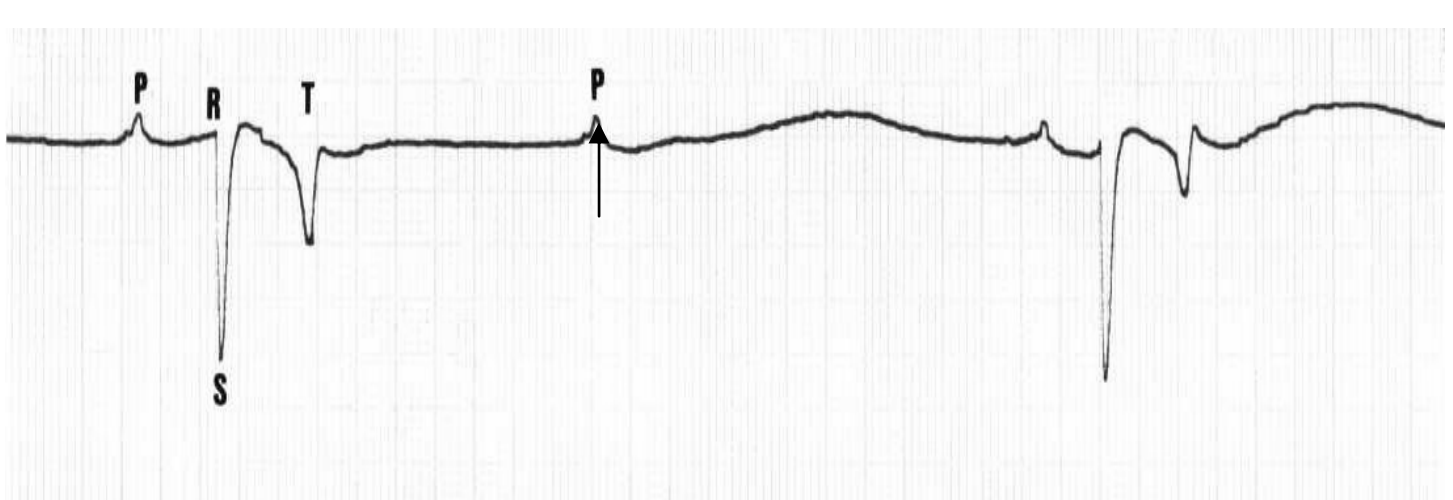

Figura 1. Traçado eletrocardiográfico de eqüino de três anos de idade, com freqüência cardíaca de $25,8 \mathrm{bpm}$, arritmia sinusal, bloqueio atrioventricular de $2^{\circ}$ grau (onda P desacompanhada de complexo QRS-seta) e derivação ápicebase.

A bradicardia sinusal presente em $1,7 \%$ dos animais, segundo Patteson (1996), é uma condição rara, que pode ser fisiológica se for abolida após excitação ou exercício, como ocorreu com o animal em questão. Entretanto, pode ser patológica e associada à baixa performance física, caso não seja abolida com o exercício.

O bloqueio sinusal ou sinus arrest foi observado em apenas um eqüino no presente estudo. Segundo Patteson (1996), esses dois ritmos não podem ser distinguidos pelo ECG, porém, considerando que sinus arrest tenha pouca freqüência em eqüinos, o bloqueio sinusal é o principal diagnóstico, sendo, normalmente, não patológico, segundo os autores. Isso foi observado no eqüino em questão, que não apresentava qualquer sinal clínico de comprometimento cardiovascular (Fig. 2).

A taquicardia sinusal, encontrada em $6,7 \%$ dos eqüinos, é considerada um ritmo freqüente, que pode ser fisiológico quando associado à dor, susto, excitação ou exercício (Fregin, 1982; Patteson, 1996). O estresse dos animais deve ser considerado, pois eles podem assustar-se com a colocação dos eletrodos e haver demora para o retorno aos valores normais. Essa pode ser considerada a provável causa de taquicardia sinusal nos eqüinos do presente estudo (Fig. 3).

Os valores médios e os erros-padrão das variáveis freqüência cardíaca, amplitude $\mathrm{e}$ duração da onda $\mathrm{P}$, duração do intervalo P-R, amplitude e duração do complexo QRS e duração do intervalo QT encontram-se na Tab. 1. 


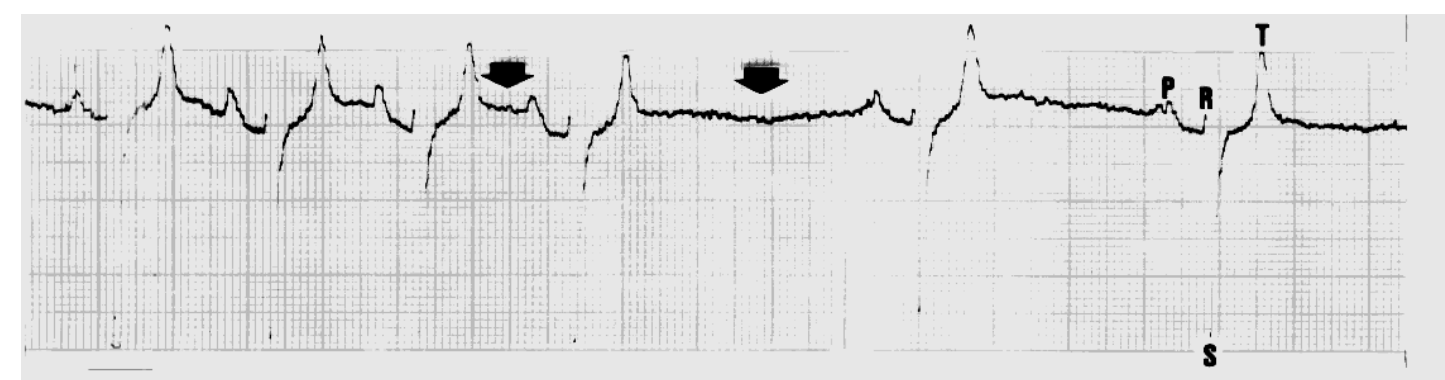

Figura 2. Traçado eletrocardiográfico de eqüino de 22 anos de idade, com freqüência cardíaca de $25 \mathrm{bpm}$, e bloqueio sinusal/sinus arrest (intervalo R-R da segunda seta apresenta-se mais que o dobro do intervalo R-R da primeira seta) na derivação ápice-base.

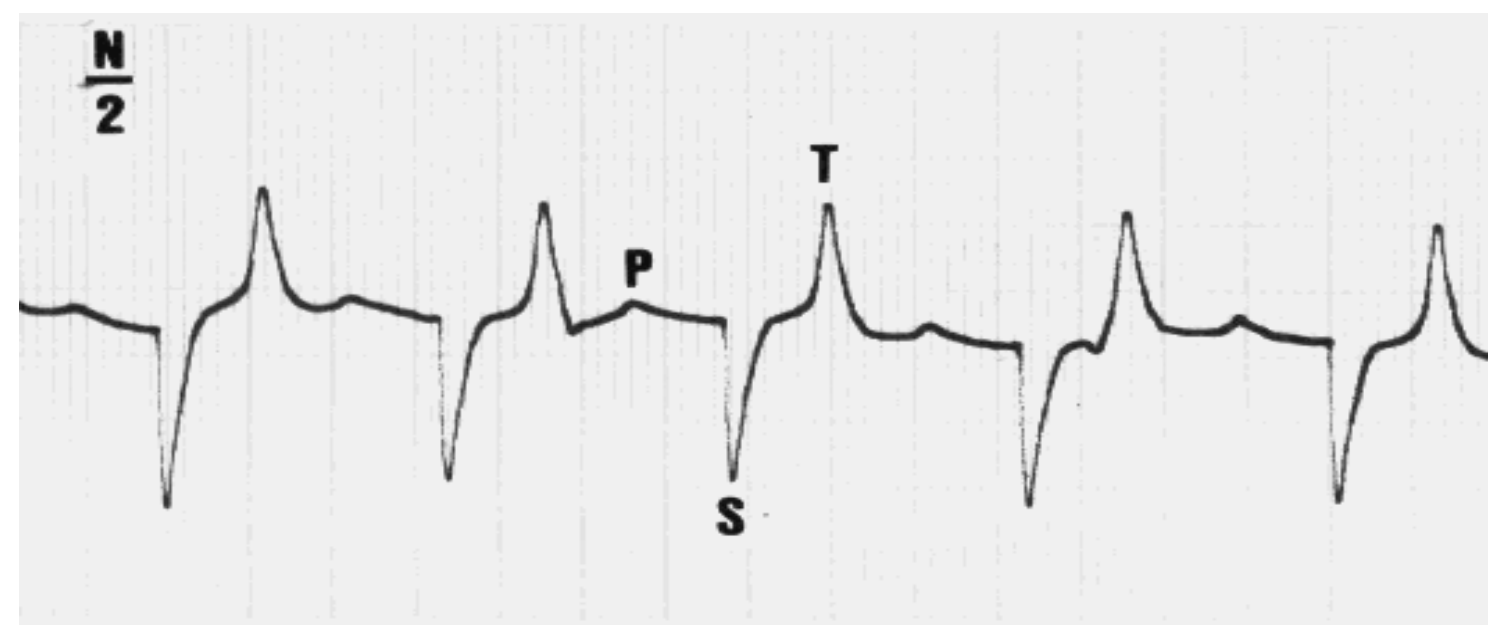

Figura 3. Traçado eletrocardiográfico de eqüino de três anos de idade, com freqüência cardíaca de $76,5 \mathrm{bpm}$ e taquicardia sinusal na derivação ápice-base.

Tabela 1. Variáveis eletrocardiográficas de 60 eqüinos da raça Mangalarga Marchador

\begin{tabular}{lcc}
\multicolumn{1}{c}{ Variável } & Média & Erro-padrão \\
\hline FC (bpm) & 42,185 & $\pm 1,657$ \\
P (s) & 0,121 & $\pm 0,004$ \\
P (mV) & 0,340 & $\pm 0,014$ \\
P-R(s) & 0,307 & $\pm 0,009$ \\
QRS (s) & 0,095 & $\pm 0,002$ \\
QRS (mV) & 0,221 & $\pm 0,027$ \\
QT(s) & 0,469 & $\pm 0,008$ \\
\hline
\end{tabular}

$\mathrm{FC}=$ freqüência cardíaca; $\mathrm{P}(\mathrm{s})=$ duração da onda $\mathrm{P}$, em segundos; $\mathrm{P}(\mathrm{mV})=$ amplitude da onda $\mathrm{P}$, em milivolts; $\mathrm{P}$ $\mathrm{R}(\mathrm{s})=$ duração do intervalo P-R, em segundos; $\mathrm{QRS}(\mathrm{s})=$ duração do complexo QRS, em segundos; $\mathrm{QRS}(\mathrm{mV})=$ amplitude do complexo QRS, em milivolts; QT(s) = duração do intervalo QT, em segundos.

A freqüência cardíaca média encontra-se dentro da faixa descrita por Hilwig (1977), que, em eqüinos adultos em repouso, pode variar de 22 a $45 \mathrm{bpm}$, isto é, a raça estudada comporta-se de maneira semelhante a outras raças já estudadas.

As ondas $\mathrm{P}$ e $\mathrm{T}$ foram observadas em $100 \%$ dos traçados. A primeira apresentou-se bífida em 41 traçados $(68,3 \%)$, única em $15(25 \%)$ e bifásica em quatro $(6,7 \%)$. $\mathrm{A}$ onda $\mathrm{T}$ foi positiva em 11 (18,3\%), negativa em $12(20 \%)$ e bifásica em 37 $(61,7 \%)$. Tal distribuição para as ondas é compatível com o padrão descrito por Patteson (1996) para essa derivação, portanto, foi semelhante à de outras raças.

Em relação à onda T, segundo Patteson (1996) e Sheard (1998), pode haver diferentes conformações nos eqüinos em repouso, até mesmo com modificações espontâneas em seu traçado, porém, isso não foi observado em nenhum dos traçados analisados. O conhecimento do padrão morfológico da onda $\mathrm{T}$ reveste-se de importância em relação ao acompanhamento do estado nutricional do 
miocárdio, revelando situações de hipóxia e infarto em eqüinos cardiopatas ou sob acompanhamento de anestesia.

Os resultados de duração encontram-se dentro dos padrões descritos por Patteson (1996) e são, em parte, similares aos resultados descritos por Ayala et al. (1994) para eqüinos da raça Andaluz, na mesma derivação bipolar ápice-base. Dessa forma, considera-se que, nas condições deste estudo, a raça não constituiu fator de variação desses parâmetros eletrocardiográficos. As raças Andaluz e Mangalarga Marchador apresentam semelhanças em suas conformações físicas, o que poderia explicar as pequenas diferenças encontradas entre elas.

As amplitudes médias das ondas Q, R, S, T+ e Tsão descritas na Tab. 2. A onda Q foi observada em 3,3\%; a R, em 73,3\% dos traçados e as S e T, em $100 \%$. O intervalo ST-T apresentou supradesnível em 41,7\% (Fig. 4). Estes resultados diferem, em parte, dos obtidos por
Ayala et al. (1994), que relataram a presença de onda $\mathrm{R}$ com amplitude média de $0,321 \mathrm{mV}$; a onda $\mathrm{S}, 1,40 \mathrm{mV}$; a T+, $0,55 \mathrm{mV}$; a T-, $0,92 \mathrm{mV}$, e o intervalo ST-T, supradesnível de $0,252 \mathrm{mV}$. Esses autores não relataram ondas $Q$ nos traçados, o que poderia ser uma particularidade dos eqüinos da raça Mangalarga Marchador.

Tabela 2. Amplitude das ondas e intervalos, encontrados no exame eletrocardiográfico de 60 eqüinos da raça Mangalarga Marchador

\begin{tabular}{lcc}
\hline Amplitude & Média & Erro-padrão \\
\hline $\mathrm{Q}(\mathrm{mV})$ & $-0,008$ & $\pm 0,006$ \\
$\mathrm{R}(\mathrm{mV})$ & 0,223 & $\pm 0,027$ \\
$\mathrm{~S}(\mathrm{mV})$ & $-2,157$ & $\pm 0,065$ \\
$\mathrm{~T}+(\mathrm{mV})$ & 0,882 & $\pm 0,055$ \\
$\mathrm{~T}-(\mathrm{mV})$ & $-1,167$ & $\pm 0,046$ \\
\hline
\end{tabular}

$\mathrm{Q}(\mathrm{mV})=$ amplitude da onda $\mathrm{Q}$, em milivolts; $\mathrm{R}(\mathrm{mV})$ $=$ amplitude da onda $\mathrm{R}$, em milivolts; $\mathrm{S}(\mathrm{mV})=$ amplitude da onda $\mathrm{S}$, em milivolts; $\mathrm{T}+(\mathrm{mV})=$ amplitude da onda $\mathrm{T}+$, em milivolts, $\mathrm{T}-(\mathrm{mV})=$ amplitude da onda T-, em milivolts.

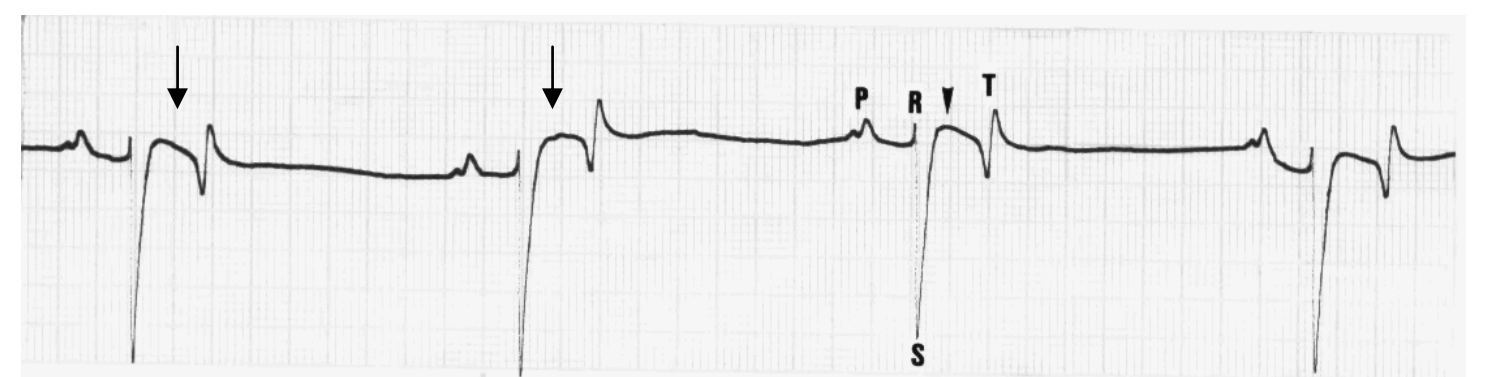

Figura 4. Traçado eletrocardiográfico de eqüino de sete anos de idade, com freqüência cardíaca de 30,6 bpm, ritmo sinusal normal e supradesnível no segmento ST-T de até $0,3 \mathrm{mV}$ (setas) na derivação ápice-base.

Em relação ao sexo (Tab. 3), observou-se diferença significativa na duração do complexo QRS e na amplitude da onda $\mathrm{S}$, aspecto não verificado por Vincenzi et al. (2000) ao estudarem a raça Mangalarga (Vincenzi et al., 2000). Uma provável explicação, também observada em outras espécies, incluindo o ser humano, seria dada pela própria diferença corpórea entre machos e fêmeas, já que essas características são mais altas nos machos.

Tabela 3. Parâmetros eletrocardiográficos de 60 eqüinos da raça Mangalarga Marchador, segundo o sexo

\begin{tabular}{lccc}
\hline \multirow{2}{*}{ Parâmetro } & Fêmea & Macho & \multirow{2}{*}{ P } \\
\cline { 2 - 3 } & Média \pm EPM. & Média \pm EPM & 0,1136 \\
Freqüência cardíaca & $44,7410 \pm 1,672$ & $39,7952 \pm 2,945$ & 0,1097 \\
Duração de P & $0,1164 \pm 0,005$ & $0,1295 \pm 0,007$ & 0,1248 \\
Amplitude de P & $0,3244 \pm 0,015$ & $0,3690 \pm 0,028$ & 0,2009 \\
Intervalo P-R & $0,2990 \pm 0,011$ & $0,3219 \pm 0,012$ & 0,0269 \\
Duração QRS & $0,0918 \pm 0,003 \mathrm{a}$ & $0,1019 \pm 0,004 \mathrm{~b}$ & 0,2859 \\
Amplitude QRS & $0,2000 \pm 0,034$ & $0,2595 \pm 0,043$ & 0,4863 \\
Intervalo QT & $0,4646 \pm 0,009$ & $0,4762 \pm 0,015$ & 0,0099 \\
Amplitude de S & $-2,0359 \pm 0,073 \mathrm{a}$ & $-2,3810 \pm 0,118 \mathrm{~b}$ & \\
\hline
\end{tabular}

EPM= erro-padrão da média.

Valores seguidos por letras distintas na linha indicam diferença significativa entre machos e fêmeas. 
Quanto à freqüência cardíaca, não houve diferença entre idades (Tab. 4), apesar de já ter sido observada diminuição dessa característica, ao longo do crescimento, em cavalos da raça Puro Sangue Inglês (Fernandes et al., 2004). A amplitude da onda $\mathrm{P}$ revelou apenas discreta diferença entre os animais mais jovens e os de até 10 anos em relação aos eqüinos com mais de 10 anos. Essa diferença, embora não estatisticamente significativa, tem importância clínica, em virtude das mudanças que ocorrem no posicionamento cardíaco dos animais mais velhos em relação aos mais jovens.

Não houve diferença no intervalo QT entre as idades. Apesar de o intervalo QT representar a repolarização ventricular e ser inversamente proporcional à freqüência cardíaca, esperava-se que a freqüência cardíaca diminuísse com a idade e o intervalo QT aumentasse, conforme resultados obtidos por Vincenzi et al. (2000) e Fernandes et al. (2004).

Tabela 4. Média e erro-padrão de parâmetros eletrocardiográficos de 60 eqüinos da raça Mangalarga Marchador, segundo a faixa etária

\begin{tabular}{lcccc}
\hline Parâmetros & 1 a 3anos & 4 a 10 anos & +10 anos & P \\
\hline FC & $46,4118 \pm 3,946$ & $42,3200 \pm 1,444$ & $38,8000 \pm 4,696$ & 0,2668 \\
P (seg) & $0,1141 \pm 0,008$ & $0,1234 \pm 0,005$ & $0,1250 \pm 0,013$ & 0,5441 \\
P (mV) & $0,2971 \pm 0,026$ & $0,33577 \pm 0,015$ & $0,4500 \pm 0,045$ & 0,0025 \\
P-R (seg) & $0,3176 \pm 0,019$ & $0,3000 \pm 0,011$ & $0,3150 \pm 0,023$ & 0,6272 \\
QRS (seg) & $0,0941 \pm 0,004$ & $0,0949 \pm 0,003$ & $0,1000 \pm 0,008$ & 0,7063 \\
QRS (mV) & $0,2286 \pm 0,033$ & $0,3521 \pm 0,035$ & $0,2667 \pm 0,039$ & 0,1100 \\
QT (seg) & $0,4447 \pm 0,019$ & $0,4800 \pm 0,008$ & $0,4700 \pm 0,025$ & 0,1451 \\
S(mV) & $-2,2059 \pm 0,128$ & $-2,0686 \pm 0,083$ & $-2,4375 \pm 0,184$ & 0,1543 \\
\hline
\end{tabular}

$\mathrm{FC}=$ freqüência cardíaca em batimentos por minuto; $\mathrm{P}(\mathrm{seg})=$ duração da onda $\mathrm{P}$, em segundos; $\mathrm{P}(\mathrm{mV})=$ amplitude da onda P, em milivolts; P-R (seg) = duração do intervalo P-R, em segundos, QRS (seg) = duração do complexo QRS, em segundos; QRS $(\mathrm{mV})=$ amplitude do complexo QRS, em milivolts;

$\mathrm{QT}(\mathrm{seg})=$ duração do intervalo QT, em segundos; $\mathrm{S}(\mathrm{mV})=$ amplitude da onda $\mathrm{S}$, em milivolts.

Não houve diferença entre idades.

\section{CONCLUSÃO}

Eqüinos da raça Mangalarga Marchador apresentam baixa freqüência de arritmias relacionadas ao excesso de esforço físico e ritmo sinusal mais freqüente, além de arritmia sinusal, sob condição de repouso e sem estarem em treinamento físico.

\section{REFERÊNCIAS BIBLIOGRÁFICAS}

AYALA, I.; MONTES, A.; FERNANDEZ del PALACIO, M.J. et al. Aportaciones al estudio electrocardiográfico del caballo. An. Vet. (Murcia), v.9, p.25-35, 1994.

AYALA, I.; MONTES, A.; BERNAL, L.J. et al. Electrocardiographic values in Spanish-bred horses in different ages. Aust. Vet. J., v.72, p.225-226, 1995.

BELGRAVE, J.O.S. A case of atrial fibrillation with congestive heart failure. Equine Vet. Educ., v.2, p.2-4, 1990.
FERNANDES, W.R.; LARSSON, M.H.M.A.; ALVES, A.L.G. et al. Características eletrocardiográficas em eqüinos clinicamente normais da raça Puro Sangue Inglês. Arq. Bras. Med. Vet. Zootec., v.56, p.143-149, 2004.

FREGIN, G.F. The equine electrocardiogram with standardized body and limb positions. Cornell Vet., v.72, p.304-324, 1982.

HILWIG, R.W. Cardiac arrhythmias in the horse. J. Am. Vet. Med. Assoc., v.170, p.153-163, 1977.

ILLERA, J.C.; ILLERA, M. Electrocardiography and heart score of horses competing in an endurance ride. Aust. Vet. J., v.64, p.88-89, 1987.

ILLERA, J.C.; ILLERA, M.; HAMLIN, R.L. Unipolar thoracic electrocardiography that induces QRS complexes of relative uniformity from male horses. Am. J. Vet. Res., v.48, p.17001702, 1987.

MITTEN, L.A. Cardiovascular causes of exercise intolerance. Vet. Clin. N. Am.: Equine Pract., v.12, p.473-494, 1996. 
PATTESON, M.W. Equine cardiology. Oxford: Blackwell Science, 1996. 254p.

RAEKALLIO, M. Long term ECG recording with holter monitoring in clinically healthy horses. Acta Vet. Scand., v.33, p.71-75, 1992.

ROBERTSON, S.A. Electrocardiography for the equine practioner. Vet. Annu., v.32, p.192-200, 1992.

ROSE, R.J.; ILKIW, J.E.; HODGDON, D. Electrocardiography, heart score and haematology of horses competing in an endurance ride. Aust. Vet. J., v.55, p.247-250, 1979.

SCHEFFER, C.J.W.; ROBBEN, J.H.; SLOET VAN OLDRUITENBORGH-OOSTERBAAN, M.M. Continuous monitoring of ECG in horses at rest and during exercise. Vet. Rec., v.137, p.371-374, 1995.

SHEARD, P.W.P. Cardiovascular system. In: COLAHAN, P.T. (Ed). Equine medicine and surgery. 5.ed. St Louis: Mosby, 1998. v.1, p.295438.
SPEIRS, V.C. Exame clínico de eqüinos. Porto Alegre: Artes Médicas, 1997. 365p.

STEWART, G.A. The heart score theory in the racehorse. Aust. Vet. J., v.57, p.422-28, 1981.

VINCENZI, R.C. Determinação dos parâmetros eletrocardiográficos de eqüinos da raça mangalarga, criados no estado de São Paulo. 1995. 73f. Dissertação (Mestrado) - Faculdade de Medicina Veterinária e Zootecnia, Universidade de São Paulo, São Paulo.

VINCENZI, R.C.; LARSSON, M.H.M.A.; FERNANDES, W.R. Parâmetros eletrocardiográficos em eqüinos clinicamente normais da raça Mangalarga. Parte III: Amplitude e duração dos complexos e intervalos. Rev. Bras. Med. Vet., v.22, p.194-198, 2000.

WHITE II, N.A.; RHODE, E.A. Correlation of electrocardiographic findings to clinical disease in the horse. J. Am. Vet. Med. Assoc., v.164, p.46-56, 1974. 